\title{
Bathymetry Estimation From Single-Frame Images of Nearshore Waves
}

\author{
Kristen D. Splinter and Robert A. Holman
}

\begin{abstract}
Existing methods for determining bathymetry from remotely sensed images of nearshore waves exploit only information on the magnitude of wavenumber $(k=2 \pi / L)$, ignoring spatial changes in wave direction $\theta$ that can provide information about bathymetry gradients. These methods also require wave period information, so they can only be used when time series imagery is available. We present an algorithm where changes in direction of refracting waves are used to determine underlying bathymetry gradients based on the irrotationality of wavenumber condition. Depth dependences are explicitly introduced through the linear dispersion relationship. The final form of the model is independent of wave period so that all necessary input measurements can be derived from a single aerial snapshot taken from a plane, unmanned aerial vehicle, or satellite. Three different methods were tested for extracting wavenumber and angle from images, i.e., two based on spatial gradients of wave phase and one based on integrated travel times between sample locations (a tomographic approach). Synthetic testing using monochromatic and bichromatic waves, with and without noise, showed that while all three methods work well under ideal wave conditions, gradient methods were overly sensitive to data imperfections. The tomographic approach yielded robust wave measurements and provided confidence limits to objectively identify unusable areas. Further tests of this method using monochromatic waves on three synthetic bathymetries of increasing complexity showed a mean bathymetry bias of $0.01 \mathrm{~m}$ and a mean $\mathrm{rms}$ error of $0.17 \mathrm{~m}$. While not always applicable, the model provides an alternative form of bathymetry estimation when celerity information is not available.
\end{abstract}

Index Terms-Image processing, sea floor, sea surface, wavelength measurement.

\section{INTRODUCTION}

$\mathbf{T}$ HE NEARSHORE region is a highly dynamic system where waves, currents, and bathymetry interact through complex feedback mechanisms. For most practical applications, these physical processes must be represented by numerical models that yield predictions of nearshore hydrodynamics when provided with input wave forcing conditions and bathymetry. Typically, wave forcing can be estimated using a number of approaches, but bathymetry, the bottom boundary condition for the models, is more difficult to obtain and changes rapidly in response to waves and storms. Thus, predictive capability is

Manuscript received October 2, 2008; revised March 3, 2009. First published June 26, 2009; current version published August 28, 2009. This work was supported under Office of Naval Research Secretary of the Navy/Chief of Naval Operations Chair of Oceanography (N00014-03-1-0973) and Coastal Geosciences programs (N00014-07-1-0490).

The authors are with the College of Oceanic and Atmospheric Sciences, Oregon State University, Corvallis, OR 97331 USA (e-mail: ksplinter@ coas.oregonstate.edu; holman@ coas.oregonstate.edu).

Color versions of one or more of the figures in this paper are available online at http://ieeexplore.ieee.org.

Digital Object Identifier 10.1109/TGRS.2009.2020157 often limited by poor knowledge of bathymetry rather than of physics.

The most accurate nearshore bathymetries are collected using traditional survey techniques in which land- and waterbased vehicles drive along a suite of survey lines, while their position is measured either using optical methods or, more recently, high-resolution kinematic global positioning system equipment (e.g., [1] and [2]). Although these methods provide an accurate [error $O$ (in centimeters)] and dense data set $[O$ (from centimeter to meter) resolution in the cross-shore and $O(25 \mathrm{~m})$ in the alongshore], they are typically very costly and time consuming and are usually restricted to fair weather conditions. They are obviously impractical for denied-access beaches of potential military interest (e.g., [3] and [4]).

Alternatively, remote sensing techniques can cover large areas and time spans at a much-reduced cost over traditional beach surveys. Light Detection And Ranging (LiDAR) is an active laser-based pulsed sensor that derives depth estimates from time-of-flight data of the optical bottom echo (e.g., [5]-[7]). Current Airborne LiDAR Bathymetry (ALB) techniques can provide accurate and dense data sets with penetration depths reaching two to three times the Secchi disk depth. New techniques using the red-channel waveforms produce accurate bathymetry measurements in the extreme nearshore $(<2 \mathrm{~m})$ [8]. However, ALB still has only limited use in many areas, particularly in midlatitudes, due to turbidity or surf-zone bubbles, and the majority of LiDAR data for the U.S. coastline have been limited to the subaerial beach on sandy coastlines. In all cases, the use of ALB requires short standoff ranges and is still not sufficiently covert for most military applications.

Hyperspectral imaging techniques exploit the wavelength dependence of optical attenuation in water to infer water depth from the color content of light reflected from the bottom (e.g., [9] and [10]). This method is also limited by water clarity (seeing the bottom) and by variability in the optical properties of both the water column and the reflecting bottom material (e.g., [10]).

Because optical penetration to the bottom is often so limiting, a series of methods has been developed that exploits observable characteristics of the sea surface to derive depth data. Plant and Holman [11] developed a shoreline-finding algorithm based on the wave dissipation maximum at the shorebreak measured from time-exposure images of wave-breaking patterns. With knowledge of the tide level and local wave conditions, the intertidal beach is mapped by defining shoreline contours as the tide level changes, creating a 3-D map of the intertidal area. Within the surf zone, Aarninkhof and Ruessink [12] found a direct relationship between modeled wave dissipation patterns 
and pixel intensity patterns seen in the time-exposure optical images. Using this proxy, model-data assimilation techniques are used to update the model bathymetry until modeled dissipation and observed dissipation proxies match within a specified range of acceptance.

Of direct relevance to this paper is a series of papers that exploits the relationship between wave frequency $\sigma$; wavelength $L=2 \pi / k$, where $k$ is the magnitude of the wavenumber; and depth $h$ represented by the linear dispersion relationship

$$
\sigma=\sqrt{g k \tanh k h}+k \cdot U
$$

where $g$ is the acceleration due to gravity, $k$ denotes the vector components of the wavenumber, and $U$ is the local current vector (which we will assume to be negligible outside the surf zone). Wave propagation is visible in many sensors (visible, infrared, and radar) so that time-space remote sensing observations can be used to measure frequency and wavenumber content for a small patch of the nearshore ocean. Depth is then estimated using (1), and a spatial map of bathymetry is built up (e.g., [3] and [13]-[18]). Linear-dispersion-based methods of bathymetry estimation are moderately accurate, with rms errors $O\left(10^{-1}-10^{0} \mathrm{~m}\right)$ (e.g., [14], [18], and [19]), and work best outside the surf zone where linear wave theory is valid (e.g., [14] and [18]-[20]).

While celerity- or linear-dispersion-based remote sensing methods can provide bathymetric measurements of reasonable accuracy, they suffer from two problems. First, they require time series data of sea-surface elevations or a proxy thereof. While snapshot data are commonly available (e.g., publicly available Google Earth images), time series in a format amenable to analysis are much more rare. Second, the linear dispersion relationship (1) exploits only the magnitude of wavenumber, not the directional variations of the vector wavenumber. Under certain conditions, wave curvature can be a very strong visual signal that can, in principle, be exploited (e.g., [3], [4], and [21]). Thus, instead of relying on the changes in just the magnitude of the wavenumber $(k=|k|)$, to invert for depth, in this paper, we wish to test the hypothesis that gradients in directional wave information $k_{x}, k_{y}$ can be used to determine gradients in local bathymetry in the absence of significant current shear, where $x$ and $y$ are the cross-shore and alongshore axes, respectively. $k_{x}$ and $k_{y}$ are defined as

$$
\begin{aligned}
& k_{x}=k \cos \theta \\
& k_{y}=k \sin \theta
\end{aligned}
$$

and $\theta$ is the local wave angle measured with respect to the crossshore axis. Of special interest, we investigate whether refractive turning can yield bathymetry estimates based simply on a single snapshot without requiring time series imagery.

The remainder of this paper is organized as follows. Section II outlines the theory and numerical implementation of the algorithm, including tests based on idealized wavenumber and wave direction data. Model sensitivity to noisy data is also discussed. Section III describes and tests three candidate methods for estimating wavenumber and wave direction data from synthetic images and further discusses algorithm sensitivity to errors in these estimates. Section IV discusses the wave period dependence of the model. Section V points out some of the complications of using real-world imagery. Finally, there are discussion and conclusion sections.

\section{Estimating Depth Gradients From DIRECTIONAL WAVE INFORMATION}

\section{A. Model Formulation}

The algorithm is based on the wave refraction equation (3) [22] that relates gradients in wavenumber $k$ and wave curvature to depth

$$
\begin{aligned}
\nabla \times k & =0 \\
\frac{\partial k_{y}}{\partial x}-\frac{\partial k_{x}}{\partial y} & =0 .
\end{aligned}
$$

Expanding (3) using (2) yields

$$
k \cos \theta \frac{\partial \theta}{\partial x}+k \sin \theta \frac{\partial \theta}{\partial y}=\cos \theta \frac{\partial k}{\partial y}-\sin \theta \frac{\partial k}{\partial x} .
$$

Depth dependence is only implicitly part of this relationship and can be made explicit by using the chain rule to substitute for spatial gradients of wavenumber

$$
\begin{aligned}
& \frac{\partial k}{\partial x}=\frac{\partial k}{\partial h} \frac{\partial h}{\partial x} \\
& \frac{\partial k}{\partial y}=\frac{\partial k}{\partial h} \frac{\partial h}{\partial y}
\end{aligned}
$$

so that the depth-dependent refraction equation becomes

$$
k \cos \theta \frac{\partial \theta}{\partial x}+k \sin \theta \frac{\partial \theta}{\partial y}=\cos \theta \frac{\partial k}{\partial h} \frac{\partial h}{\partial y}-\sin \theta \frac{\partial k}{\partial h} \frac{\partial h}{\partial x} .
$$

Equation (6) is rearranged so that the unknown bathymetry gradients $(\partial h / \partial x$ and $\partial h / \partial y)$ are a function of wave curvature (the curl of the directional component of wavenumber) and wavenumber

$$
\frac{\partial h}{\partial x} \sin \theta-\frac{\partial h}{\partial y} \cos \theta=-\kappa\left(\frac{\partial \sin \theta}{\partial x}-\frac{\partial \cos \theta}{\partial y}\right)
$$

where

$$
\kappa=\frac{k}{\partial k / \partial h} .
$$

With the exception of $\kappa$, all terms in (7) are directly observable from a single image. However, the term $\partial k / \partial h$ would seem to depend on wave period $T$, so it requires temporal sampling or alternate estimation means. In the following, the performance of the algorithm will be examined based on the assumption that wave period information is available. However, in Section IV, it will be demonstrated that a universal form for $\kappa$ can be found that is independent of wave period. Thus, bathymetry can be estimated from snapshot images, albeit by solving a much more complex form of the equation.

Equation (7) was originally implemented in finite-difference form as a tridiagonal matrix and inverted to estimate depth on a row-by-row basis since there were still two unknowns. Knowing the wave period, $\kappa$ was approximated numerically 
TABLE I

Summary of SynthetiC BeACH PARAmeters UsEd IN TESTING ThE REFRACTION-BASED BATHYMETRY ESTIMATION MODEL

\begin{tabular}{cccccc}
\hline \hline Case & $\begin{array}{c}\text { Grid size } \\
{[\mathrm{nx}, \mathrm{ny}]}\end{array}$ & $\begin{array}{c}d x, d y \\
(\mathrm{~m})\end{array}$ & $\beta_{x}$ & $\begin{array}{c}\text { Period) } \\
(\mathrm{s})\end{array}$ & $\begin{array}{c}\theta_{0} \\
\text { degrees }\end{array}$ \\
\hline Planar & $250 \times 250$ & 2 & $1: 100$ & 8 & 30 \\
Shoal & $250 \times 250$ & 2 & $1: 100$ & 8 & 30 \\
Pocket & $250 \times 500$ & 2 & $1: 100$ & 8 & 0 \\
\hline
\end{tabular}

using a first-order finite-difference implementation of the linear dispersion relationship (1) based on the variations in depth $h$ for small deviations $( \pm \epsilon)$ of $k$. Several limitations were found with the use of (7). The first was that an initial depth was required at some offshore location in order to use the finitedifference technique. As well, under locally normal $(\theta=0)$ wave incidence, the inversion was poorly conditioned, and solutions were unstable. The first of these problems was inherent to the differential equation. To remedy the second problem, the model was rearranged, using (5) to replace $\partial h / \partial y$ with

$$
\frac{\partial h}{\partial y}=\left(\frac{\partial k / \partial y}{\partial k / \partial x}\right) \frac{\partial h}{\partial x} .
$$

Solving for cross-shore gradients in depth independently as a function of local gradients in directional wave information

$$
\beta_{x}=\frac{\partial h}{\partial x}=\frac{\kappa\left(\frac{\partial \cos \theta}{\partial y}-\frac{\partial \sin \theta}{\partial x}\right)}{\sin \theta-\cos \theta \frac{\partial k}{\partial y} / \frac{\partial k}{\partial x}} .
$$

This form of the model is numerically more stable for low wave angles, provided that the denominator is nonzero. Another advantage of (10) is that the solutions at different alongshore locations are independent of each other and the calculated cross-shore gradients can be filtered using a $3 \sigma$ median filter to remove anomalies, as will be discussed further in the following. Depths are then estimated by integrating (10) in the cross-shore from a location of known depth, such as the shoreline. We chose to integrate depths from an interior point to minimize errors associated with end points. Since integration proceeds away from an initial value, any error early in the integral is carried throughout the domain, and total errors will be large. Thus, different choices of initial values (offshore or onshore) could yield completely different error estimates. Nevertheless, comparisons are included since bathymetry is the ultimate desired product and since error maps provide insight into the nature of the errors. The remainder of this paper will focus on the performance of the algorithm based on (10).

\section{B. Algorithm Performance for Idealized Data}

We first tested the accuracy of the model using idealized directional wave information. Tests were carried out on three synthetic bathymetries: a planar beach, a planar beach with a superimposed Gaussian shoal to add 2-D variable bathymetry, and a pocket beach with headlands at either end (see Figs. 7 and 8 for reference). Beach parameters are summarized in Table I.

Wavenumbers for each domain were determined from the linear dispersion relationship (1) based on known bathymetry.
TABLE II

RMS ERROR OF $\beta_{x}$ AND $h$ FOR IDEALIZED DATA

\begin{tabular}{lrrr}
\hline \hline & & \multicolumn{2}{c}{ Beach } \\
& Planar & Shoal & Pocket \\
\hline$\beta_{x}$ & $8.8 \times 10^{-6}$ & $8.8 \times 10^{-6}$ & $9.6 \times 10^{-5}$ \\
$h(m)$ & $2.9 \times 10^{-3}$ & $1.4 \times 10^{-3}$ & $3.7 \times 10^{-3}$ \\
\hline \hline
\end{tabular}

TABLE III

RMS ERROR OF $k, \theta, \beta_{x}$, AND $h$ FOR NOISE SENSITIVITy TESTS

\begin{tabular}{lrrrr}
\hline \hline Test \# & $k\left(\mathrm{~m}^{-1}\right)$ & $\theta\left(^{\circ}\right)$ & $\beta_{x}$ & $h(\mathrm{~m})$ \\
\hline 1 & $\mathrm{~N} / \mathrm{A}$ & 0.2 & 0.02 & 0.15 \\
2 & 0.002 & N/A & 0.03 & 2.0 \\
\hline \hline
\end{tabular}

Wave angle was solved for using (4) by stepping shoreward from the known offshore wave angle $\theta_{0}$ using the spatial changes in wavenumber to determine changes in wave angle. The resulting data were then used in (10) and the process inverted to solve for bathymetry gradients $\beta_{x}$ and, by integration, the estimated bathymetry $h$. Model performance is summarized in Table II. Since the solution was just the inverse of the equations used to generate the idealized data, it was not surprising that the model performed exceptionally well for all cases tested. RMS errors in $\beta_{x}$ were $<1 \%$ of the true gradients, and rms errors in bathymetry were typically a few millimeters.

\section{Algorithm Performance for Noisy Data}

We performed two different tests using the planar beach case in which Gaussian white noise was added to $k$ and $\theta$ in order to test the model's sensitivity to data errors. To start, true $k$ and true $\theta$ were calculated, as described in Section II-B. In test 1 , we tested the model's sensitivity to errors in $\theta$ or wave curvature with ideal wavenumber data by applying Gaussian white noise of $1 \%$ of the median $\theta$ value to the true $\theta$. True $k$ values were used with the noisy $\theta$ estimates to solve for $\beta_{x}$. In test 2 , we tested the model's sensitivity to errors in $k$, thus testing that if our estimates in wavenumber were off but curvature could be measured more robustly, could the model still converge to the correct solution. This was done by applying Gaussian white noise of $1 \%$ of the median $k$ value to the true $k$ and using the noisy $k$ values with true $\theta$ values in (10) to solve for $\beta_{x}$. Algorithm performance is summarized in Table III.

Examination of the sensitivity results leads to several conclusions. First, results in Table III show a high sensitivity to even this low level of noise. The primary reason for this is that the noise introduced in the data $(k, \theta)$ affects the algorithm through its spatial gradients $(\partial k / \partial x$, etc.), so that, for the $2-\mathrm{m}$ grid spacing of the tests, even the small rms noise levels used produced gradients that were on the order of $200 \%(k)$ and $150 \%(\theta)$ of the true gradient signals. Second, the model's sensitivity to gradients implies that the method that is eventually selected for estimating these input variables from images will need to be spatially smooth, either through an explicit smoothing or implicitly due to the method. Third, even extreme noise in the beach slope estimates (rms error in $\beta_{x}>$ true $\beta_{x}$ ) yielded substantially smaller errors in bathymetry since the slope gradient errors were Gaussian and were partially averaged out by integration. 




Fig. 1. Example of a shoaling wave field for a planar bathymetry. The crossshore distance is along the vertical axis, and the shoreline is at the bottom. The wave period and offshore wave angle were $8 \mathrm{~s}$ and $30^{\circ}$, respectively. Surface elevation is measured in meters.

\section{BATHyMETRY EStimation From Synthetic IMAGES}

Results in the previous section were based on idealized $k$ and $\theta$ information that was given a priori. Typically, these variables will have to be estimated from images of shoaling and refracting waves. In this section, we describe and test three candidate methods using synthetic idealized data.

\section{A. Methods for Extracting Directional Wave Information From Images}

In general, a wave field $\eta(x, y)$ can be represented in terms of spatially variable amplitude and phase functions $A(x, y)$ and $\phi(x, y)$, respectively, as

$$
\eta(x, y)=\Re\left(A(x, y) e^{i \phi(x, y)}\right)
$$

where the enclosed function is called the analytical signal and $\Re()$ represents the real-valued component thereof. The phase function $\phi(x, y)$ is related to the components of wavenumber $\left(k_{x}, k_{y}\right)$ by

$$
\phi(x, y)=\int_{0}^{x} k_{x} d x+\int_{0}^{y} k_{y} d y .
$$

If the spatial map of phase is given or can be found from image data, this relationship can be inverted to find $k_{x}$ and $k_{y}$ as the $x$ and $y$ spatial derivatives of phase.

Sea surface elevations (Fig. 1) or proxies such as changes in image intensity caused by sloping wave surfaces are real valued, and the phase function cannot be directly extracted from the signal. Instead, phase function estimates are found by taking the Hilbert transforms in the cross-shore direction of the observed data to get the analytic signal $\hat{\eta}$ [23], [24]. To reduce spectral leakage, the input data are windowed using a $20 \%$ cosine taper, with the tapered points being removed from later analysis. The phase function $\phi(x, y)$ is then determined by

$$
\phi(x, y)=\tan ^{-1}\left(\frac{\Im(\hat{\eta}(x, y))}{\Re(\hat{\eta}(x, y))}\right)
$$


Fig. 2. (Top) Phase estimate (in radians) of the input wave field (Fig. 1). (Bottom) Unwrapped phase (in radians) starting at the top-left corner of the image and integrating in both directions.

where $\Im()$ and $\Re()$ indicate the imaginary and real components, respectively, and the phase function $\phi(x, y)$ is measured in radians. The resulting 2 -D phase map has values between $-\pi$ and $\pi$ [Fig. 2 (top)], with discontinuities in each time phase jumping from $+\pi$ to $-\pi$. By unwrapping the phase function in both directions, a global phase map $\phi_{G}(x, y)$ [Fig. 2 (bottom)] is produced, in which simple gradient methods can be directly applied to estimate the two wavenumber components.

1) Gradient Methods for Determining Directional Wave Information: Two different methods for determining wavenumber through phase gradients are tested. Eventually, both are abandoned in favor of a method that implicitly smoothes estimates across the domain. Discussion is nevertheless included, both for completeness and to illustrate the importance of smooth input data. In the first method, wavenumber components are computed by taking the spatial gradients of the globally unwrapped phase $\phi_{G}(x, y)$ [Fig. 2 (bottom)] through finite differencing

$$
\left[k_{x}, k_{y}\right]=\nabla \phi_{G}(x, y)
$$

For the case of a monochromatic wave field (Fig. 1), gradients of the globally unwrapped phase provide accurate directional wavenumber information (Table IV).

For more complicated seas, however, the performance of the global gradient method degrades substantially. Fig. 3 shows the group structure of waves due to a bichromatic wave field. 
TABLE IV

MeAn (RMS) Error of $k, \theta, \beta_{x}$, AND $h$ Estimations For Monochromatic (M), Noise Sensitivity (N), and Bichromatic (B) WaVe Conditions, Using the Three Data Extraction TeChniques. While Wavenumber Varies Across the Domain, a Representative Magnitude for Comparison Is $0.1-0.4 \mathrm{~m}^{-1}$. The True $\beta_{x}$ Is 0.01

\begin{tabular}{llrrrrrrrr}
\hline \hline & Case & \multicolumn{2}{c}{$k\left(\mathrm{~m}^{-1}\right)$} & \multicolumn{2}{c}{$\theta\left(^{\circ}\right)$} & \multicolumn{1}{c}{$\beta_{x}$} & \multicolumn{2}{c}{$h(\mathrm{~m})$} \\
\hline \multirow{3}{*}{$\mathrm{M}$} & $\nabla \phi_{G}(x, y)$ & $5.1 \times 10^{-4}$ & $\left(1.3 \times 10^{-3}\right)$ & -0.03 & $(0.2)$ & $5.6 \times 10^{-6}$ & $\left(3.5 \times 10^{-3}\right)$ & 0.03 & $(0.06)$ \\
& $\nabla \phi(x, y)$ & $-3.8 \times 10^{-3}$ & $\left(5.9 \times 10^{-3}\right)$ & 0.3 & $(0.3)$ & $8.7 \times 10^{-5}$ & $\left(4.4 \times 10^{-3}\right)$ & 0.03 & $(0.06)$ \\
& $\mathrm{PHH} 08$ & $4.3 \times 10^{-4}$ & $\left(5.0 \times 10^{-3}\right)$ & -0.1 & $(0.2)$ & $6.0 \times 10^{-4}$ & $\left(1.7 \times 10^{-3}\right)$ & -0.02 & $(0.10)$ \\
\hline \multirow{2}{*}{$\mathrm{N}$} & $\nabla \phi_{G}(x, y)$ & $3.9 \times 10^{-3}$ & $\left(3.4 \times 10^{-2}\right)$ & -0.3 & $(12.2)$ & $3.5 \times 10^{-3}$ & $(1.64)$ & 1.33 & $(26.02)$ \\
& $\nabla \phi(x, y)$ & $-2.2 \times 10^{-4}$ & $\left(2.3 \times 10^{-2}\right)$ & -0.3 & $(11.8)$ & $6.2 \times 10^{-4}$ & $(2.33)$ & -0.66 & $(41.23)$ \\
& $\mathrm{PHH} 08$ & $2.8 \times 10^{-4}$ & $\left(5.4 \times 10^{-3}\right)$ & -0.1 & $(0.2)$ & $5.5 \times 10^{-4}$ & $\left(1.3 \times 10^{-3}\right)$ & -0.01 & $(0.06)$ \\
\hline \multirow{2}{*}{$\mathrm{B} \phi_{G}(x, y)$} & $4.1 \times 10^{-1}$ & $\left(4.3 \times 10^{-1}\right)$ & 6.9 & $(40)$ & $4.1 \times 10^{-3}$ & $(0.44)$ & -0.30 & $(4.37)$ \\
& $\nabla \phi(x, y)$ & $-4.8 \times 10^{-4}$ & $\left(1.3 \times 10^{-2}\right)$ & 0.3 & $(0.7)$ & $2.1 \times 10^{-3}$ & $(0.01)$ & -0.03 & $(0.48)$ \\
& PHH08 & $2.5 \times 10^{-3}$ & $\left(4.6 \times 10^{-3}\right)$ & -0.1 & $(0.2)$ & $6.0 \times 10^{-4}$ & $(0.01)$ & -0.09 & $(0.49)$ \\
\hline \hline
\end{tabular}

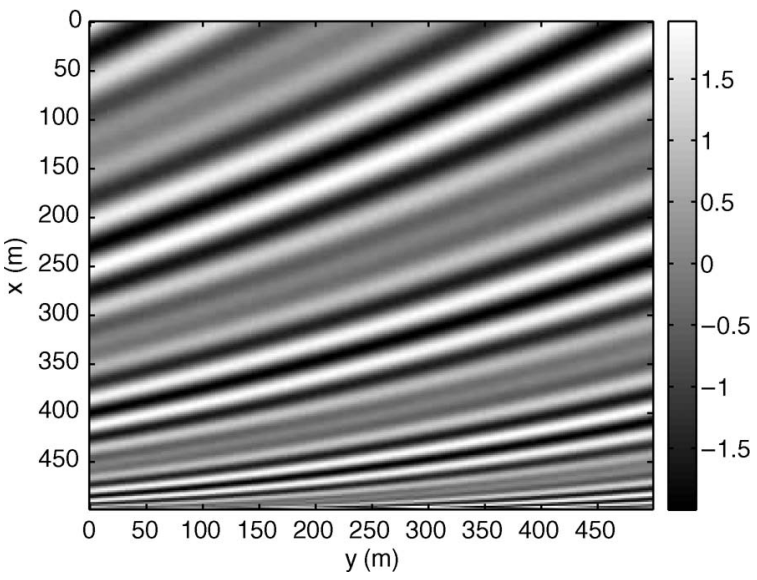

Fig. 3. Example of a narrow-banded wave field with two waves, i.e., $T_{1}=8 \mathrm{~s}$ and $T_{2}=10 \mathrm{~s}$. The offshore wave angle is $30^{\circ}$ for both cases. Surface elevation is measured in meters.

The wrapped phase [Fig. 4 (top)] includes anomalies at the nodal points that cause two problems. First, attempts to unwrap the phase in two dimensions [Fig. 4 (bottom)] are nonunique and include jumps in phase that cause anomalies in the estimated wavenumber. Second, since waves on either side of nodal points are in antiphase, the wavelength of the waves that are split across the group nodes are correspondingly split and appear as short waves [Fig. 4 (top)].

The former problem can be solved by removing the requirement for phase unwrapping. Therefore, the second method that we test is a local gradient method that computes gradients in the complex domain space of the analytic signal [25], [26]

$$
\left[k_{x}, k_{y}\right]=\nabla \phi(x, y)=\Re\left[\frac{\nabla \hat{\eta}(x, y)}{i \hat{\eta}(x, y)}\right]
$$

This method produces accurate estimates of $k$ for most of the images. In areas of wave group nodes, wavenumber components are higher than expected due to the extra phase shift of $\pi$. Wavenumbers that lie outside $3 \sigma$ from the mean $k$ are replaced with the local mean wavenumber. Estimating $k$ from local gradients is an improvement over the global phase unwrapping technique when applied to finite-bandwidth waves because it isolates the areas (wave group nodes) where wavenumber estimates are not consistent with the expected values.


Fig. 4. (Top) Phase estimate (in radians) of the finite-bandwidth wave field. (Bottom) Two-dimensional unwrapped phase (in radians) starting from the top left of the image. Jumps in phase that are less than $2 \pi$ are not unwrapped and cause the streaks in the unwrapped phase image.

From the wavenumber estimates, wave angle is calculated from the arctangent of the directional wave components

$$
\theta=\tan ^{-1}\left(\frac{k_{y}}{k_{x}}\right)
$$

2) Nonlinear Inversion Technique: A third technique for estimating $k$ and $\theta$ from images uses a nonlinear inversion method [27], hereinafter referred to as PHH08. The method, essentially a tomographic approach based on wave propagation time delays across many spatial lags, uses a nonlinear search to find the single values of wavenumber and direction that best explain the complex analytical signal, found by the Hilbert transform of the imagery, over each of a suite of subregions 


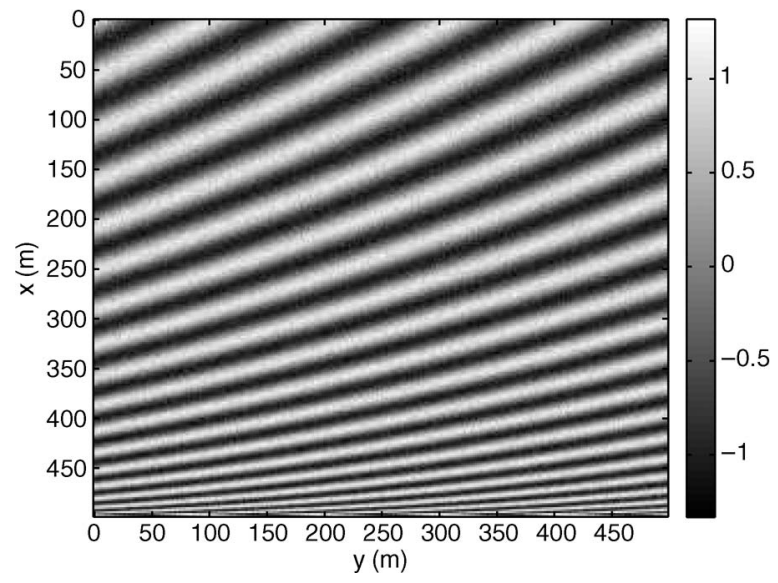

Fig. 5. Example of a monochromatic wave field with $10 \%$ noise superimposed. $T=8 \mathrm{~s}$. The offshore wave angle is $30^{\circ}$. Surface elevation is measured in meters.

of the domain. Because time delays for all possible pixel pairs (close and widely spaced) in each subregion are incorporated in the estimate, extreme contamination by short gradient noise is avoided. The shortest resolvable features that can be estimated from variations in $k$ are equal to about ten times the local water depth [27], so the spatial resolution of the model domain is such that it will not attempt to estimate variations in $k$ and $\theta$ that are shorter than these scales. For the cases tested, estimates for wavenumber and wave angle are done on a coarse tomographic domain with spatial resolution of $30 \mathrm{~m}$, utilizing input data $\pm 60 \mathrm{~m}$ in the cross-shore and $\pm 100 \mathrm{~m}$ in the alongshore direction to estimate $k$ and $\theta$. The data are then interpolated onto a finer grid with spatial resolution of $2 \mathrm{~m}$. These both speed up the inversion technique and remove high-frequency noise that may be in the initial data signal. The coarse domain, however, is not well suited to the extreme nearshore where rapid variations in wavenumber and angle may occur that are unresolved by the spatial scale of the tile. Note also that since the resulting data will be used in gradient calculations, linear interpolation will yield stepwise changes in gradients, hence patchy bathymetry gradients, so higher order interpolations must be used. The reader is referred to [27] for a detailed description of the method.

\section{B. Synthetic Test Results}

1) Comparison of Data Extraction Techniques: The three methods of data extraction were tested using synthetic waves approaching the planar beach. Three tests were carried out: a monochromatic case (M) of 8-s waves approaching with a $30^{\circ}$ offshore angle of incidence (Figs. 1 and 2), a noiseless bichromatic sea (B) with 8- and 10-s waves approaching from $30^{\circ}$ (Figs. 3 and 4), and a noisy case (N) of the same waves with superimposed Gaussian white noise with standard deviation equal to $10 \%$ of the maximum wave height (Figs. 5 and 6). For each case, maps of $k, \theta, \beta_{x}$, and $h$ were computed by each of the three data extraction methods and compared to known values through both mean and rms statistics. Results are summarized in Table IV.

All methods performed very well for the monochromatic wave field. The Hilbert transform introduced slight ringing into


Fig. 6. (Top) Phase estimate (in radians) of the noisy wave field. (Bottom) Two-dimensional unwrapped phase (in radians) starting from the top left of the image.

the analytic signal that carried through into the phase estimates. The gradient methods for determining $k$ and $\theta$ emphasized this ringing, with rms errors in $k$ being on the order of a few percent. $\beta_{x}$ oscillated around the true mean, causing larger rms errors in $\beta_{x}$ for the gradient methods but reasonable estimates for integrated bathymetry $h$ [Fig. 7 (top)]. However, the performance of the gradient methods declined rapidly with data imperfections. Phase jumps in the bichromatic case yielded unacceptable results of $k$ and $\theta$ for the global phase unwrapping method, although they were better handled by the local gradient method. RMS errors in $h$ were at least an order of magnitude greater in the bichromatic case using the gradient methods. As expected, the addition of white noise to the data led to performance statistics for the gradient methods that were completely unusable. Early attempts at spatial filtering improved results somewhat, but a general robust methodology was never found. Thus, gradient methods are not recommended for the purpose of wave characteristic estimation.

The PHH08 approach performed well for all three tests. Since this method uses a constant depth assumption over each analysis subarray, slight biases in $k$ and $\theta$ on the order of $1 \%$ were expected for the bathymetry and model domain used. For the monochromatic case, the PHH08 approach had error results similar to that of the gradient methods, with rms errors in $k$ being on the order of a few percent. The rms error in $\beta_{x}$ was on 



Fig. 7. (Solid) True bathymetry and (dash) estimated bathymetry measured in meters. (Top) $k$ and $\theta$ extracted from the image using global phase unwrapping methods. The mean error in the estimated bathymetry was $3 \mathrm{~cm}$. (Bottom) $k$ and $\theta$ extracted from the image using PHH08 techniques. The mean error in the estimated bathymetry was $-2 \mathrm{~cm}$.

the order of $1 \%$, and the rms error in integrated bathymetry was $10 \mathrm{~cm}$ [Fig. 7 (bottom)]. The small amount of ringing associated with computation of the Hilbert transform that led to oscillations in $\beta_{x}$ estimates using gradient methods was smoothed over with the PHH08 approach. Errors using this method were concentrated near the shoreline, where rapid changes in $k$ were likely not well resolved on the coarse tomographic domain, and at the lateral boundaries, where a reduced number of points used in the estimation led to less accurate results. The latter issue can be resolved by overlapping images in the alongshore and/or removing the edge points.

In contrast to gradient methods, the performance of the PHH08 method was robust to noise, as expected, with rms statistics for all parameters being similar to those of the monochromatic case. For the bichromatic case, the method was still troubled at the wave node locations, but since this approach provided a skill estimate, values with skill $<0.5$ were objectively identified, removed, and interpolated over for a more accurate solution. No such skill estimate was available for the gradient methods. RMS errors in $\beta_{x}$ for the bichromatic case were on the order of the true beach slope, producing larger errors in integrated bathymetry (rms error in $h \sim 0.5 \mathrm{~m}$ ). As a whole, the PHH08 method produced the most consistent results, with skill estimates to objectively identify areas of concern. Thus, the PHH08 method is preferred for $k$ and $\theta$ extraction and will be used for the remainder of this paper and for recommended future work.

2) Complex Bathymetry Results: The PHH08 method was utilized to extract $k$ and $\theta$ data for monochromatic wave conditions on more complex bathymetry. The results are summarized in Table V. For the planar beach with a Gaussian shoal, bathymetry gradients over the shoal were well predicted [Fig. 8(a)], with mean (rms) errors in bathymetry being on the order of $1 \mathrm{~cm}$ $(10 \mathrm{~cm})$. For the pocket beach, normal wave incidence, combined with $\partial k / \partial y=0$, produced an unstable solution for $\beta_{x}$ at some locations in the cross-shore around $y=500 \mathrm{~m}$. Since this is a known limitation of the refraction model, a standard deviation filter was applied to remove anomalies and smooth over these areas. Integrated bathymetry had slight offsets at the center of the image and close to shore where changes in wavenumber and angle were not well resolved due to the tiling method of the PHH08 model [Fig. 8(b)]. Even with these known limitations, mean (rms) errors in bathymetry were on the order of $1 \mathrm{~cm}(30 \mathrm{~cm})$. Overall, the results were very encouraging that the proposed model could predict complex bathymetry based on refraction signatures.

\section{WAVE PeRiod DePendence}

The aforementioned analysis was based on the use of (10). All terms in this equation are directly observable from a single image with the exception of $\kappa$. Wave period cannot be estimated from a snapshot, unless it spans into deepwater, where $T=$ $\sqrt{2 \pi L_{o} / g}$. It would appear that motion imagery (a sequence of images) is required and that single snapshots are not helpful. In fact, $\kappa$ is independent of wave period. For example, in the shallow water limit $(k h<\pi / 10), \kappa$ simplifies to eliminate the wave period dependence as follows:

$$
\begin{aligned}
k & =\frac{\sigma}{\sqrt{g h}} \\
\frac{\partial k}{\partial h} & =-\frac{\sigma}{\sqrt{g h}} \frac{1}{2 h} \\
\kappa & =\frac{k}{\partial k / \partial h}=-2 h .
\end{aligned}
$$

Fig. 9 shows the deviation from $\kappa=-2 h$ as a function of nondimensional depth $(k h)$. At the small $k h$ values associated with shallow water, the value of $-\kappa / 2 h$ is just 1.0 , as expected earlier. However, even for larger values of $k h$, the curve is independent of wave period, so the algorithm can be applied to a single snapshot.

The form of the curve in Fig. 9 can be well approximated by a polynomial, such that

$\kappa=-2 h\left[0.55(k h)^{3}-0.61(k h)^{2}+0.55(k h)+0.91\right]$.

Substituting (18) into (10) yields a somewhat complicated form that must be solved numerically but is based solely on wavenumber and wave angle estimates that can be extracted from a single snapshot. 
TABLE V

MEAN (RMS) ERRoR FOR THE PHH08 METHOD OVER COMPLEX BATHYMETRY

\begin{tabular}{|c|c|c|c|c|c|c|c|c|}
\hline ase & & 1) & & $\left({ }^{\circ}\right)$ & & & & (m) \\
\hline toal & $.6 \times 10^{-4}$ & $(5.3 \times$ & 0.13 & $(0.4$ & $6.55 \times 10^{-2}$ & $(2.0$ & 0.01 & .13) \\
\hline cket & $3.2 \times 10^{-4}$ & $\left(8.9 \times 10^{-3}\right)$ & 0.01 & $(0.06)$ & $6.5 \times 10^{-4}$ & $\left(8.6 \times 10^{-3}\right)$ & -0.01 & $(0.28)$ \\
\hline
\end{tabular}


Fig. 8. (Solid) True bathymetry and (dash) estimated bathymetry using (10). (Top) Beach with shoal: The mean error in bathymetry was $-0.01 \mathrm{~m}$. (Bottom) Pocket beach: The mean error in the estimated bathymetry was $-0.01 \mathrm{~m}$.



Fig. 9. Expected error associated with the assumption of shallow water $\kappa=-2 \mathrm{~h}$ versus nondimensional depth $(k h)$.

\section{Application to Field Data}

This paper was motivated by the need for remote sensing methods to measure bathymetry and the common availability of remotely sensed snapshots in which strong wave refraction signals must be related to underlying bathymetry gradients. While our eyes are good at picking out the desired pattern, a variety of problems complicate the computer automation of this process.

Optically, images contain contamination at many scales. At low wavenumber, lighting varies both in the skydome and due to the general variation of water surface reflectivity with vertical viewing angle. There are also complications in the region between nonbreaking and breaking waves where the optical signature of a wave crest shifts from being dark (nonbreaking) to light (breaking). Since the optical signature of a wave depends on its local wave steepness [28], short but steep features, such as wind chop, are exaggerated compared to the slowly varying swell.

Filtering techniques, such as weighted standard deviation and bandpass filters, can be used to isolate and enhance the swell band to a reasonable degree [29]. Better yet, the PHH08 method does a good job estimating a dominant $k-\theta$ over a userselected analysis window, averaging out both short- and longscale contamination and smoothing through anomalies. The PHH08 model also returns skill estimates for each $k-\theta$ estimate, so questionable results are easily identified and possibly eliminated. However, it should be recognized that the refractionbased algorithm proposed in this paper is useful only in cases of narrow-banded swell with strong refraction patterns.

\section{DISCUSSION}

The motivation for this paper was to develop a bathymetry estimation technique that could be based on single-image snapshots rather than time series imagery and that also exploited both the magnitude and varying directions of wavenumber. Thus, it was rewarding to see that the equations could be formulated without reference to wave period. However, the method does not work under all conditions. For example, the solution blows up under the joint circumstances of normal incidence, relative to bathymetry contours, and no alongshore gradients in wavenumber, when the denominator of (10) approaches zero. In this case, there is simply no refractive signal to exploit.

The method is also based on an underlying assumption of a monochromatic or very narrow-banded wave field that can be represented adequately by a single wavenumber and direction. For time series imagery data, more complicated seas could be handled spectrally by isolating individual coherent components of the wave field by both frequency and direction. However, for single snapshots, such a partitioning is not possible, and the method will yield noisy results. Fortunately, the PHH08 method flags the quality of results through a skill statistic that can be used to objectively identify where bathymetry estimates can and cannot be trusted.

The primary products of the refraction method are estimates of bathymetry gradients, in contrast to linear-dispersion-based methods that directly estimate depth. Consequently, depth estimates must be found by integration from some known value, for example, the shoreline. In some parts of the region of interest, bathymetry gradient estimates may be poor, perhaps due to locally low wave contrast or the aforementioned problem of normal incidence with no alongshore gradients. Cross-shore integration cannot continue through regions such as these that are flagged as bad, so knowledge of the full profile can be limited. However, these regions are usually spatially patchy and can be bypassed using the surrounding information. The details of such an algorithm are beyond the scope of this paper. 
The effect of currents on the dispersion relation has been neglected in the current model. The implications of this simplification are discussed here. Uniform currents that are perpendicular to the direction of wave propagation have no effect on the frequency and wavenumber estimates determined from (1). Alternatively, currents that are parallel or at an angle to the direction of wave propagation will affect the estimated wave phase speed $c$ and frequency $\sigma$, as seen by a stationary observer, but have no effect on the estimated wavenumber, provided that the current can be considered uniform over the area in question [30]. Thus, it is the spatial variation (shear) in the current that can contaminate the bathymetric refraction patterns of incident waves. The magnitude of this error can be found by considering the simplified case of a wave propagating in the $x$-direction against an opposing current in shallow water. The equation governing wave angle [22, pp. 106] can be written as

$$
\frac{\partial \theta}{\partial x}=\frac{-1}{c_{a}} \frac{\partial c_{a}}{\partial y}
$$

where

$$
c_{a}=\sqrt{g h}-U .
$$

Therefore, changes in wave angle are related to the combined contributions of bathymetric changes and currents

$$
\frac{\partial \theta}{\partial x}=\frac{-1}{\sqrt{g h}-U}\left(\frac{1}{2}\left(\frac{g}{h}\right)^{1 / 2} \frac{\partial h}{\partial y}-\frac{\partial U}{\partial y}\right) .
$$

For an example of a 1-m/s rip current with a half-width of $50 \mathrm{~m}$ in $1 \mathrm{~m}$ of water $(\partial U / \partial y=-1 / 50)$, the terms on the righthand side become

$$
\begin{aligned}
\frac{-1}{\sqrt{g h}-U} & =\frac{-1}{\sqrt{9.81}-1} \\
\frac{1}{2}\left(\frac{g}{h}\right)^{1 / 2} \frac{\partial h}{\partial y}-\frac{\partial U}{\partial y} & =\frac{\sqrt{g}}{2} \frac{\partial h}{\partial y}-\frac{1}{50} .
\end{aligned}
$$

The first term suggests that strong opposing currents reduce $\partial \theta / \partial x$ by $30 \%$, while the second term suggests that the shears of a strong rip current can produce refractive turning that is equivalent to bathymetry gradients of $\sim 1 / 80$. The sense of the error would be to reduce the estimated depths in a rip channel.

In the nearshore, the currents with the strongest shears are either longshore currents or rip currents. In the former case, the direction of flow will be nearly orthogonal to the usual near-normal angles of wave approach, so contamination will be minimal. As shown previously, rip currents can cause a refractive turning that is equivalent to an $\sim 1 / 80$ slope, but the error will be predominantly in longshore slope, smoothing out rip channels as opposed to cross-shore beach slope. Areas of strong shear around tidal inlets should, of course, be avoided with this method. With the assumption that all changes in wavenumber are due to bathymetric variation, the presence of a current, such as a rip current or flow from an inlet, will change the wavenumber and wave angle characteristics and yield errors in bathymetry gradient estimates. In the most extreme cases, such as strong rip currents exiting the surf zone in a rip channel, the proposed method will predict a shoal in the location of the current, while the bathymetry is most likely a trench. Since this method is most applicable outside the surf zone, these situations should be limited. In tidally influenced areas, images taken at the peaks of the tidal cycle (high tide/low tide) when tidal currents are reduced should be used to limit error in bathymetry gradient estimation.

Initial tests of the refractive method were based on gradient approaches for estimating the required wavenumber and direction inputs. While successful for perfect (synthetic) data, they were sensitive to noise, particularly the high wavenumber noise due to short wind-blown chop that is typical of real-world images. Extensive filtering was required to produce stable results. Thus, it was not until the introduction of the tomographic method of PHH08 that smooth and reliable values of wave quantities could be derived for imperfect data. Because time delays for all possible pixel pairs (close and widely spaced) in the subregion are incorporated in the estimate, extreme contamination by short gradient noise is avoided. Areas of known wave anomalies such as the edge of the surf zone where the optical signatures of wave fronts change from dark to white breakers can be reasonably smoothed through. For waves with a finitebandwidth spectrum, a single best-fit set of wave characteristics can be found. A requirement of this approach is computation of a corresponding confidence interval or skill for the solution. For some cases, waves will simply be too broadbanded for representation by a single direction and wavelength. These can be identified objectively by low skill values and the data disregarded.

As with other remote sensing methods of determining bathymetry, the refraction-based method has limitations in its application. Most importantly, the model requires that there be measurable gradients in both wavenumber and angle. Although longer waves feel the bottom at greater depth, $(k h$ for any given depth is always less for longer waves), shorter period waves have their advantage in the nearshore because they undergo rapid changes in wave angle and wavenumber at shallower depths (e.g., [22, pp. 108]). This suggests that the method is best suited for shorter period swell conditions in intermediate $(\pi / 10<k h<\pi)$ water depths.

\section{CONCLUSION}

An algorithm has been developed to estimate nearshore bathymetry based on the changing directions of refracting waves. The model uses an augmented form of the refraction equation that relates gradients in bathymetry to gradients in $k$ and $\theta$ through the chain rule. The equations can be cast in a form that is independent of wave period, so it can be solved using wavenumber and direction data from a single snapshot rather than the normally required time series of images.

Three methods for extracting $k$ and $\theta$ data from images were tested. Under monochromatic conditions, all methods performed well. However, for cases with high-frequency noise or a nonideal wave field, the two gradient methods for determining $k$ and $\theta$ were found to be unusable. The tomographic approach of the PHH08 method was robust to these complications and had the added advantage of providing skill estimates that allowed objective identification of unacceptable results. Synthetic testing of the model using monochromatic waves on three bathymetries of increasing complexity showed that the 
model accurately estimated 2-D bathymetry gradients, hence bathymetry, with a mean bias of $0.01 \mathrm{~m}$ and a mean rms error over the three beaches of $0.17 \mathrm{~m}$. While the model is not useful for cases of complex seas or small refraction signals, the simplified data requirement of only a single snapshot is attractive. The model is perhaps best suited for shorter period swell conditions, for example, from a semi-enclosed sea, where strong refraction patterns are visible and $k$ and $\theta$ are easily extracted from a single-frame image.

\section{ACKNOWLEDGMENT}

The authors would like to thank N. Plant for his insightful discussions, technical assistance, and the use of his nonlinear model employed in this paper. They would also like to thank J. Stanley for his technical assistance. K. Splinter would also like to thank P. Catalan, J. Long, M. Haller, T. Özkan-Haller, and M. Palmsten for the many discussions and reviews along the way. The authors would also like to thank the three anonymous reviewers for their comments and recommendations in improving this paper.

\section{REFERENCES}

[1] W. A. Birkemeier and C. Mason, "The CRAB: A unique nearshore surveying vehicle," J. Surv. Eng., vol. 110, no. 1, pp. 1-7, Mar. 1984.

[2] J. H. MacMahan, "Hydrographic surveying from a personal watercraft," J. Surv. Eng., vol. 127, no. 1, pp. 12-24, 2001.

[3] W. W. Williams, "The determination of gradients of enemy-held beaches," Geogr. J., vol. 107, pp. 76-93, Jul. 1946.

[4] H. R. Seiwell, "Military oceanography in World War II," Mil. Eng., vol. 39, no. 259, pp. 202-210, 1947

[5] G. D. Hickman and J. E. Hogg, "Application of an airborne pulsed laser for near-shore bathymetric measurements," Remote Sens. Environ., vol. 1, no. 1, pp. 47-58, Mar. 1969.

[6] G. C. Guenther, Airborne Laser Hydrography: System Design and Performance Factors. Rockville, MD: NOAA, Mar. 1985. NOS, NOAA, Professional Paper Series.

[7] J. L. Irish and W. J. Lillycrop, "Scanning laser mapping of the coastal zone: the SHOALS system," ISPRS J. Photogramm. Remote Sens., vol. 54, no. 2/3, pp. 123-129, Jul. 1999.

[8] S. Pe'eri and W. Philpot, "Increasing the existence of very shallow-water LIDAR measurements using the red-channel waveforms," IEEE Trans. Geosci. Remote Sens., vol. 45, no. 5, pp. 1217-1223, May 2007.

[9] J. C. Sandidge and R. J. Holyer, "Coastal bathymetry from hyperspectral observations of water radiance," Remote Sens. Environ., vol. 65, no. 3, pp. 341-352, Sep. 1998.

[10] S. M. Adler-Golden, P. K. Acharya, A. Berk, M. W. Matthew, and D. Gorodetzky, "Remote bathymetry of the littoral zone from AVIRIS, LASH, and QuickBird imagery," IEEE Trans. Geosci. Remote Sens., vol. 43, no. 2, pp. 337-347, Feb. 2005.

[11] N. Plant and R. Holman, "Intertidal beach profile estimation using video images," Mar. Geol., vol. 140, no. 1/2, pp. 1-24, 1997.

[12] S. Aarninkhof and B. Ruessink, "Video observations and model predictions of depth-induced wave dissipation," IEEE Trans. Geosci. Remote Sens., vol. 42, no. 11, pp. 2612-2622, Nov. 2004.

[13] P. S. Bell, "Shallow water bathymetry derived from an analysis of X-band marine radar images," Coast. Eng., vol. 37, no. 3/4, pp. 513-527, Aug. 1999.

[14] H. Stockdon and R. Holman, "Estimation of wave phase speed and nearshore bathymetry from video imagery," J. Geophys. Res., vol. 105, no. C9, pp. 22 015-22 033, 2000.

[15] J. P. Dugan, C. Piotrowski, and J. Z. Williams, "Water depth and surface current retrievals from airborne optical measurements of surface gravity wave dispersion," J. Geophys. Res., vol. 106, no. C8, pp. 16903-16915, Aug. 2001.

[16] C. Piotrowski and J. Dugan, "Accuracy of bathymetry and current retrievals from airborne optical time-series imaging of shoaling waves," IEEE Trans. Geosci. Remote Sens., vol. 40, no. 12, pp. 2602-2612, Dec. 2002.
[17] S. K. Misra, A. Kennedy, and J. Kirby, "An approach to determining nearshore bathymetry using remotely sensed ocean surface dynamics," Coast. Eng., vol. 47, no. 3, pp. 265-293, Jan. 2003.

[18] C. M. Senet, J. Seemann, S. Flampouris, and F. Ziemer, "Determination of bathymetric and current maps by the method DiSC based on the analysis of nautical X-band radar image sequences of the sea surface (Nov. 2007)," IEEE Trans. Geosci. Remote Sens., vol. 46, no. 8, pp. 2267-2279, Aug. 2008.

[19] S. Flampouris, F. Ziemer, and J. Seemann, "Accuracy of bathymetric assessment by locally analyzing radar ocean wave imagery (Feb. 2008)," IEEE Trans. Geosci. Remote Sens., vol. 46, no. 10, pp. 2906-2913, Oct. 2008.

[20] T. K. Holland, "Application of the linear dispersion relation with respect to depth inversion and remotely sensed imagery," IEEE Trans. Geosci. Remote Sens., vol. 39, no. 9, pp. 2060-2072, Sep. 2001.

[21] W. Munk and M. Traylor, "Refraction of ocean waves: A process linking underwater topography to beach erosion," J. Geol., vol. 55, pp. 1-26, 1947.

[22] R. G. Dean and R. A. Dalrymple, Water Wave Mechanics for Engineers and Scientists, vol. 2. Singapore: World Scientific, 1991.

[23] J. S. Bendat and A. G. Piersol, Random Data: Analysis and Measurement Techniques, 2nd ed. New York: Wiley-Interscience, 1986.

[24] J. P. Havlicek, J. W. Havlicek, and A. C. Bovik, "The analytic image," in Proc. IEEE Int. Conf. Image Process., Oct. 26-29, 1997, pp. 446-448.

[25] J. P. Havlicek, D. S. Hardin, and A. C. Bovik, "The multi-component AM-FM image representation," IEEE Trans. Image Process., vol. 5, no. 6, pp. 1094-1100, Jun. 1996.

[26] J. P. Havlicek, J. W. Havlicek, N. D. Mamuya, and A. C. Bovik, "Skewed 2D Hilbert transforms and computed AM-FM models," in Proc. IEEE Int. Conf. Image Process., 1998, pp. 602-606.

[27] N. Plant, K. T. Holland, and M. Haller, "Ocean wavenumber estimation from wave-resolving time series imagery," IEEE Trans. Geosci. Remote Sens., vol. 46, no. 9, pp. 2644-2658, Sep. 2008.

[28] R. Walker, Marine Light Field Statistics, ser. Wiley series in pure and applied optics, J. Goodman, Ed. New York: Wiley, 1994.

[29] K. D. Splinter and R. A. Holman, "Bathymetric estimation based on wave refraction patterns," in Proc. 30th Int. Conf. Coastal Eng., J. M. Smith, Ed. Singapore: World Scientific, 2006, vol. 1, pp. 451-463.

[30] I. A. Svendsen, Introduction to Nearshore Hydrodynamics, ser. Advanced Series on Ocean Engineering, vol. 24. Singapore: World Scientific, 2006.



Kristen D. Splinter received the B.Sc.(Hons.) degree in civil engineering from Queen's University, Kingston, ON, Canada, in 2002, the M.S. degree in coastal and oceanographic engineering from the University of Florida, Gainesville, in 2004, and the Ph.D. degree in geological oceanography, with certificate in marine resource management, from Oregon State University, Corvallis.

She held Natural Sciences and Engineering Research Council of Canada student research appointments at Queen's University in 2000 and 2001, as well as a student research appointment at Abteilung von Wasserversorgung und Entsorgung von Abwasser, Universität Essen, Essen, Germany, in 2002. Her current research interests include remote sensing of the nearshore ocean, coastal processes, and morphological evolution of the nearshore environment.



Robert A. Holman received the B.S. degree in mathematics and physics from the Royal Military College of Canada, Kingston, ON, Canada, in 1972 and the Ph.D. degree in physical oceanography from Dalhousie University, Halifax, NS, Canada, in 1979.

$\mathrm{He}$ then joined the College of Oceanic and Atmospheric Sciences, Oregon State University, Corvallis, where he is currently a Full Professor. $\mathrm{He}$ is also a Secretary of the Navy/Chief of Naval Operations Chair of Oceanography. He is also the Head of the Argus Program for nearshore remote sensing. His research interest includes the application of optical remote sensing methods to understand the dynamics of the nearshore ocean. 